\title{
A family of optimal termination detection algorithms
}

\author{
Neeraj Mittal · S. Venkatesan · Sathya Peri
}

Received: 28 January 2005 / Accepted: 20 April 2007 / Published online: 30 June 2007

(C) Springer-Verlag 2007

\begin{abstract}
An important problem in distributed systems is to detect termination of a distributed computation. A computation is said to have terminated when all processes have become passive and all channels have become empty. In this paper, we present a suite of algorithms for detecting termination of a non-diffusing computation for an arbitrary communication topology under a variety of conditions. All our termination detection algorithms have optimal message complexity. Furthermore, they have optimal detection latency when message processing time is ignored.
\end{abstract}

Keywords Monitoring distributed system - Termination detection - Arbitrary communication topology - Optimal algorithm · Diffusing and non-diffusing computations . Simultaneous and delayed initiations $\cdot$ Single-hop and multi-hop application messages

\section{Introduction}

One of the fundamental problems in distributed systems is to detect termination of an ongoing distributed computation. The problem arises, for example, when computing shortest paths between pairs of nodes in a network. The distributed computation is modeled as follows. A process can either be

A preliminary version of the paper first appeared in the 18th Symposium on Distributed Computing (DISC), 2004 [27].

N. Mittal $(\bowtie) \cdot$ S. Venkatesan $\cdot$ S. Peri

Department of Computer Science, The University of Texas at Dallas, Richardson, TX 75083, USA

e-mail: neerajm@utdallas.edu

S. Venkatesan

e-mail: venky@utdallas.edu

S. Peri

e-mail: sathya.p@student.utdallas.edu in active state or passive state. Only an active process can send an application message. An active process can become passive at anytime. A passive process becomes active only on receiving an application message. A computation is said to have terminated when all processes have become passive and all channels have become empty. The problem of termination detection was independently proposed by Dijkstra and Scholten [11] and Francez [12] more than two decades ago. Since then, many researchers have worked on this problem and, as a result, a large number of algorithms have been developed for termination detection (e.g., [4,9,10,14$16,21,23-26,29,30,32])$. Note that termination is a stable property. Thus a simple approach for detecting termination is to repeatedly take a consistent snapshot of the underlying computation using any of the algorithms described in $[1,5,13,18]$, and then test the snapshot for the termination condition. More efficient algorithms have been developed which do not depend on taking consistent snapshots of the computation. Most of the termination detection algorithms can be broadly classified into four categories, namely computation tree based, invigilator based, double wave based and single wave based.

In the computation tree based approach, a dynamic tree is maintained based on the messages exchanged by the underlying computation. A process not currently "participating" in the computation, on receiving an application message, remembers the process that sent the message (and joins the dynamic tree) until it "leaves" the computation. This creates a parent-child relationship among processes that are currently "part" of the computation. A process may join and leave the tree many times. Example of algorithms based on this idea can be found in $[11,30,4]$.

In the invigilator based approach, a distinguished process called the coordinator, is responsible for maintaining current status of all processes either directly or indirectly. The 
coordinator may either explicitly maintain the number of processes that are currently "participating" in the computation or may only know whether there exists at least one process that is currently "participating" in the computation (ascertained via missing credit/weight $[15,24]$ or some other mechanism [21]). Many algorithms in this class assume that the topology contains a star and the coordinator is directly connected to every process $[15,24]$. These algorithms can be generalized to work for any communication topology at the expense of increased message complexity.

The next two classes of algorithms are based on the notion of wave [31]. A wave is a control message or a subset of control messages that sweep through the entire system visiting all processes on the way. As the wave travels through processes, it collects their local snapshots, which are then combined to obtain a snapshot of the entire system.

In the double wave based approach, two (possibly inconsistent) snapshots of the computation are taken in such a way that there is at least one consistent snapshot lying between the two snapshots. The interval between the two snapshots is then tested for any possible activity. In case the interval is quiescent (no activity took place), termination can be announced. It can be proved that evaluating the termination condition for either of the snapshots is actually equivalent to evaluating the condition for any consistent snapshot lying between the two snapshots [2]. Various algorithms differ in the manner in which they test for quiescence of an interval and emptiness of channels. Examples of algorithms based on this idea can be found in [9, 14,23,25].

In the single wave based approach, a snapshot of the computation is first tested for consistency. If the test evaluates to true, then the snapshot is analyzed for the termination condition. The consistency test is such that if the snapshot is not consistent then the test will definitely evaluate to false. It is possible that the test may evaluate to false even if the snapshot is consistent. However, if the snapshot is taken after the computation has terminated, then the test is guaranteed to evaluate to true. Various algorithms differ in the manner in which they test for consistency of a snapshot and emptiness of channels. Examples of algorithms based on this idea can be found in $[16,23,29]$.

In addition, termination detection algorithms can also be classified based on two other attributes: (1) whether the distributed computation starts from a single process or from multiple processes (diffusing computation versus non-diffusing computation), and (2) whether the detection algorithm should be initiated along with the computation or can be initiated anytime after the computation has started (simultaneous initiation versus delayed initiation). Delayed initiation is useful when the underlying computation is message-intensive and therefore it is preferable to start the termination detection algorithm later when the computation is "close" to termination.
Table 1 shows the (worst-case) message complexity and detection latency for the best algorithm in each of the four classes and for our algorithms. The table also indicates assumptions, if any, made about the communication topology. The complexity expressions given in the table are derived under the assumptions that: (1) application messages are only exchanged between neighboring processes in the topology, and (2) message transmission time is $O(1)$ unit and message processing time is ignored. (The latter assumption is only used to analyze the detection latency of a termination detection algorithm and not to prove its correctness.) Most termination detection algorithms are analyzed under these two assumptions (e.g., $[11,23,4,8])$. Henceforth, in this paper, all complexity expressions are presented or derived under the above two assumptions unless otherwise stated. Later, in Section 3.6, we present a more detailed analysis of the detection latency of our termination detection algorithms in terms of both message processing time and message transmission time. When message processing time is considered explicitly, it turns out that our termination detection algorithms are no longer latency-optimal for all communication topologies (but are still messageoptimal).

In [21], Mahapatra and Dutt consider the case when application messages can be exchanged between arbitrary processes. For some algorithms in parallel computing, even non-neighboring processes may be required to exchange application messages with each other [7]. Later, in Section 5, we describe how to maintain optimality of our termination detection algorithm when application messages may travel multiple hops.

Chandy and Misra [6] prove that any termination detection algorithm, in the worst case, must exchange at least $M$ control messages, where $M$ is the number of application messages exchanged. Also, in the worst-case, the detection latency of any termination detection algorithm measured in terms of message hops is $D$, where $D$ is the diameter of the communication topology. Algorithms derived from the computation tree based approach typically have optimal message complexity but non-optimal detection latency (e.g., [11, 17]). On the other hand, algorithms that use the invigilator based approach typically have optimal detection latency but non-optimal message complexity (e.g., $[15,24,21]$ ). (The message-complexity is optimal only when the diameter of the communication topology is constant.) To our knowledge, at present, there is no termination detection algorithm that has optimal message complexity as well as optimal detection latency for all communication topologies. The message complexity of a termination detection algorithm measures the overhead imposed by the algorithm on the system during its execution. Its detection latency measures the delay incurred between when the computation terminates and when the termination is actually detected (and announced). Clearly, 
Table 1 Comparison of various termination detection algorithms (assume diffusing computation, simultaneous initiation and single-hop application messages unless indicated otherwise)

\begin{tabular}{|c|c|c|c|}
\hline Termination detection algorithm & Message complexity & Detection latency $^{\mathrm{a}}$ & Communication topology \\
\hline Computation tree based (e.g., [11]) & $O(M)$ & $O(N)$ & Any \\
\hline Invigilator based (e.g., [24]) & $O(M)$ & $O(1)$ & Diameter is constant \\
\hline Modified invigilator based ${ }^{\text {b }}$ (e.g., [24]) & $O(M D)$ & $O(D)$ & Any \\
\hline Double wave based ${ }^{\mathrm{c}}$ (e.g., [2]) & $O(M N)$ & $O(D)$ & Any \\
\hline Single wave based ${ }^{c}$ (e.g., [23]) & $O(M N)$ & $\overline{O(D)}$ & Any \\
\hline Our algorithm & $\underline{O(M)}$ & $\underline{O(D)}$ & Any \\
\hline Our algorithm (non-diffusing computation) & $\underline{O(M+N)}$ & $\underline{O(D)}$ & Any \\
\hline \multicolumn{4}{|l|}{ Our algorithm (non-diffusing computation } \\
\hline and delayed initiation) & $O(\bar{M}+E)$ & $\underline{O(D)}$ & Any \\
\hline \multicolumn{4}{|l|}{ Our algorithm (non-diffusing computation } \\
\hline and multi-hop application messages) & $O(M H+N)$ & $O(D)$ & Any \\
\hline
\end{tabular}

$N$ number of processes in the system, $E$ number of channels (or links) in the communication topology, $M$ number of application messages exchanged by the underlying computation, $\bar{M}$ number of application messages exchanged by the underlying computation after the termination detection algorithm began, $D$ diameter of the communication topology, $H$ average number of hops traveled by application messages, $\underline{O(\cdot)}$ complexity expression is optimal

${ }^{a}$ Ignoring message processing time

${ }^{\mathrm{b}}$ Invigilator based adapted for arbitrary communication topology

${ }^{\mathrm{c}}$ Wave is collected using a breadth-first-search spanning tree to ensure optimality of detection latency

it is desirable to minimize both message complexity and detection latency of a termination detection algorithm.

Note that, for a general non-diffusing computation, any termination detection algorithm must exchange at least $N-1$ control messages in the worst-case, where $N$ is the number of processes in the system. Chandrasekaran and Venkatesan [4] prove another lower bound on message-complexity: if the termination detection algorithm is initiated anytime after the computation has started, then the algorithm, in the worst case, must exchange at least $E$ control messages, where $E$ is the number of communication channels (or links) in the topology. They also show that delayed initiation is not possible unless all channels are first-in-first-out (FIFO).

Our contributions in the paper are as follows. We present three message-optimal and latency-optimal termination detection algorithms for arbitrary communication topologies under varying assumptions such as (1) whether the initiation is simultaneous or delayed and (2) whether application messages are single-hop or multi-hop. Our first algorithm assumes that the initiation is simultaneous and application messages are single hop. Our second algorithm, which is derived from the first algorithm, assumes that the initiation may be delayed but application messages are single-hop. Our third algorithm, which is again derived from the first algorithm, assumes that the initiation is simultaneous but application messages may be multi-hop. A message-optimal and latency-optimal termination detection algorithm for the case when initiation may be delayed and application messages may be multi-hop can be obtained by combining the modifications used for second and third algorithms. All our termination detection algorithms have very low message overhead as well. Specifically, a message has to carry only one integer whose maximum value is bounded by $2 D$, which is independent of the number of messages exchanged by the underlying computation. Intuitively, we achieve optimality with respect to message-complexity and detection-latency at the same time by combining computation tree based and invigilator based approaches.

The paper is organized as follows. In Sect. 2, we discuss the system model and notation used in this paper, and describe the termination detection problem. Section 3 describes an optimal termination detection algorithm for the case when the detection algorithm has to be initiated along with the computation. Section 4 describes the modifications required to handle the case when the detection algorithm can be initiated at any time after the computation has commenced. Section 5 describes the modifications required to handle the case when application messages can be exchanged between arbitrary processes. Finally, we present our conclusion and outline directions for future research in Sect. 6.

\section{System model and problem statement}

\subsection{Model and notation}

We assume an asynchronous distributed system consisting of $N$ processes $P=\left\{p_{1}, p_{2}, \ldots, p_{N}\right\}$, which communicate with each other by exchanging messages over a communication network. There is no common clock or shared memory. 
Processes are non-faulty and channels are reliable. Message delays are finite but may be unbounded.

We do not assume that the underlying communication topology is fully connected. Two processes can communicate directly with each other only if they are neighbors in the topology. If two processes are neighbors in the topology, then we say that there is a channel between them. We assume that all channels are bidirectional. We use $E$ to refer to the number of channels in the communication topology.

Processes execute events and change their states. A local state of a process, therefore, is given by the sequence of events it has executed so far starting from the initial state. Events are either internal or external. An external event could be a send event or a receive event. An event-internal or externalcauses the local state of a process to be updated. In addition, an external event causes a message to be sent (send event) or received (receive event).

Events on a process are totally ordered. However, events on different processes are only partially ordered by the Lamport's happened-before relation [19], which is defined as the smallest transitive relation satisfying the following properties:

1. if events $e$ and $f$ occur on the same process, and $e$ occurred before $f$ in real time then $e$ happened-before $f$, and

2. if events $e$ and $f$ correspond to the send and receive, respectively, of a message then $e$ happened-before $f$.

A snapshot of the system is a collection of local states, one from each process. A local state of a process can be captured by the set of events that have been executed so far on that process. (An empty set of events denotes the initial state.) Therefore, in terms of events, a snapshot, which is also referred to as a cut, is a set of events satisfying the following property:

\section{$S$ is a snapshot $\triangleq$}

$\langle\forall e, f: e$ and $f$ are on the same process :

$(e \rightarrow f) \wedge(f \in S) \Rightarrow e \in S\rangle$

We say that a snapshot passes through an event if it is the last event on that process to be contained in the snapshot. A snapshot that contains the receive event of a message but not its send event is not a valid snapshot of the system. Such a snapshot is called an inconsistent snapshot. Conversely, we say that a snapshot (or cut) is consistent if the following holds:

$S$ is a consistent snapshot $\triangleq$

$$
\langle\forall e, f::(e \rightarrow f) \wedge(f \in S) \Rightarrow e \in S\rangle
$$

Next, we formally define the termination detection problem.

\subsection{The termination detection problem}

The termination detection problem involves detecting when an ongoing distributed computation has terminated. The distributed computation is modeled as follows. A process can be either in an active state or a passive state. A process can send a message only when it is active. An active process can become passive at anytime. A passive process becomes active on receiving a message. The computation is said to have terminated when all processes have become passive and all channels have become empty.

To avoid confusion, we refer to the messages exchanged by the underlying computation as application messages, and the messages exchanged by the termination detection algorithm as control messages. Unless indicated otherwise, we describe our termination detection algorithms assuming that application messages are only exchanged between neighboring processes, that is, application messages are single-hop. This is consistent with the assumption made by most termination detection algorithms (e.g., [4, 11, 17,23]). Later, in this paper, we discuss the case when application messages may be exchanged between arbitrary processes, that is, when application messages are multi-hop.

In this paper, when a process sends a control message, we distinguish between two cases-whether the process has created the message itself or is simply forwarding the message it has received from a neighboring process to another neighboring process. In the former case, we say that the process has generated the control message. Further, we refer to the messages in the latter case as derivative control messages.

It is desirable that the termination detection algorithm exchange as few control messages as possible, that is, the algorithm has low message complexity. The higher the message complexity of a termination detection algorithm, the higher the overhead imposed by it on the system during execution. Clearly, the overhead imposed by a termination detection algorithm should be minimized. Further, once the underlying computation terminates, the algorithm should detect it as soon as possible, that is, the algorithm has low detection latency [21]. For computing detection latency, it is typically assumed that each message hop takes at most one time unit and message processing time is negligible [3,31]. Finally, the amount of control information carried by any message - application or control-is minimal, that is, the algorithm has low bit-message complexity.

A computation is said to be diffusing if only one process is active initially; otherwise it is non-diffusing. If the termination detection algorithm has to be initiated along with the computation, then we refer to it as simultaneous initiation. On the other hand, if the termination detection algorithm can be initiated anytime after the computation has started, then we refer to it as delayed initiation. 


\section{An optimal algorithm for simultaneous initiation}

In this section, we first describe the main idea behind our algorithm, and then give its formal description. Later, we prove the correctness of our algorithm and also show that it is message-optimal and latency-optimal. Our approach is based on combining computation tree based and invigilator based approaches. This allows us to achieve the best of both approaches, namely optimal message-complexity of computation tree based approach and optimal detection latency of invigilator based approach.

\subsection{The main idea}

We first describe the main idea behind the algorithm assuming that the underlying computation is a diffusing computation. We relax this assumption later.

\subsubsection{Detecting termination of a diffusing computation}

First, we briefly explain the main idea behind the computation tree based and the invigilator based approaches. Then we discuss how to combine them to obtain the optimal algorithm.

Computation tree based approach: Consider a termination detection algorithm using computation tree based approach $[4,11]$. Initially, only one process, referred to as the initiator, is active and all other processes are passive. A process, on receiving an application message, sends an acknowledgment message to the sender as soon as it knows that all activities triggered by the application message have ceased. The initiator announces termination as soon as it has received an acknowledgment message for every application message it has sent so far and is itself passive. The algorithm has optimal message complexity because it exchanges exactly one control message, namely the acknowledgment message, for every application message exchanged by the underlying computation. The detection latency, however, is far from optimal. Specifically, a chain of pending acknowledgment messages (hereafter, referred to as an acknowledgment chain) may grow to a length as long as $M$, where $M$ is the number of application messages exchanged by the underlying computation. (The reason is that a process may appear multiple times on an acknowledgment chain as is the case with the algorithm of [4].)

The detection latency of the algorithm can be reduced from $O(M)$ to $O(N)$ (assuming $M=\Omega(N)$ ) as follows [11]. Suppose a process has not yet sent an acknowledgment message for an application message it received earlier. In case the process receives another application message, it can immediately send an acknowledgment message for the latter application message. For termination detection purposes, it is sufficient to assume that all computation activities triggered by the receipt of the latter application message are triggered by the former application message. We refer to the former application message as an engaging application message and to the latter as a non-engaging application message.

Observe that the set of engaging application messages imposes a parent-child relationship among processes "currently participating" in the computation. Specifically, if a process is active or has not yet received an acknowledgment message for every application message it has sent so far, then it is "currently a part" of the computation and is referred to as a non-quiescent process. Otherwise, it is "not currently a part" of the computation and is referred to as a quiescent process. At any time, the computation tree, which is dynamic, consists of the set of processes that are non-quiescent at that time.

Invigilator based approach: Now, consider a termination detection algorithm using the invigilator based approach [21]. (The algorithm described here is actually a simplified version of the algorithm presented in [21] but, nevertheless, captures the main idea.) One process is chosen to act as the coordinator. The coordinator is responsible for maintaining the current status of all processes in the system either directly or indirectly. Suppose a process receives an application message. In case the coordinator does not already know that it is currently active, it sends a control message indicating "I am now active" to the coordinator. Once the process knows that the coordinator has received the control message, it sends an acknowledgment message to the sender of the application message. On the other hand, if the process has already informed the coordinator that it is currently active, then it immediately acknowledges the application message. Once a process becomes passive and has received an acknowledgment message for every application message it has sent so far, it sends a control message indicating "I am now passive" to the coordinator. Intuitively, if the underlying computation has not terminated, then, as per the coordinator, at least one process is currently active. When the coordinator is directly connected to every process in the system, the algorithm has optimal message complexity (at most three control messages for every application message) and optimal detection latency (which is $O(1)$ ). When the topology is arbitrary, however, for communication between the coordinator and other processes, a static breadth-first-search (BFS) spanning tree rooted at the coordinator has to be constructed. Every control message that a process sends to the coordinator (along the BFS spanning tree) may cause up to $D$ additional (or derivative) control messages to be exchanged, thereby increasing the message complexity to $O(M D)$.

Achieving the best of the two approaches: As explained above, in the computation-tree based approach, a process reports its status, when it becomes quiescent, to its parent. 
On the other hand, in the invigilator based approach, a process reports its status, when it becomes quiescent, to the coordinator (directly or indirectly). The main idea is to restrict the number of times processes report their status to the coordinator - to achieve optimal message complexity - and, at the same time, restrict the length of an acknowledgment chain-to achieve optimal detection latency.

Whenever a process reports its status to the coordinator, as many as $D$ control messages may have to be exchanged. As a result, to achieve optimal message complexity, the number of times when processes report their quiescent status to the coordinator should be bounded by $O(M / D)$. The rest of the time processes should report their quiescent status to their respective parents in the computation tree. To ensure optimal detection latency, the length of an acknowledgment chain should be bounded by $O(D)$. The main problem is to determine, while the computation is executing, when a process should choose the former over the latter. In our algorithm, a process, by default, is supposed to report its status to its parent until it learns that the length of a chain of pending acknowledgment messages, starting from it, has become sufficiently long, that is, the length of the chain has become $\Theta(D)$. At that time, it starts reporting its status to the coordinator. Specifically, it first sends an st_active message signifying that "my computation subtree is currently active" to the coordinator. It waits until it has received an acknowledgment from the coordinator in the form of an ack_st_active message. The receipt of the ack_st_active message implies that the coordinator is aware of some activity in the system and therefore will not announce termination as yet. It then sends an acknowledgment message to its parent, thereby breaking the link with its parent and shortening the acknowledgment chain. Later, when it becomes quiescent, it sends an st_passive message indicating "my computation subtree has now become passive" to the coordinator.

To measure the length of an acknowledgment chain, we piggyback an integer counter (referred to as hop counter) on every application message that represents the current length of an acknowledgment chain. On receiving an application message, if a process learns that the length of the acknowledgment chain has become at least $D$, then it resets the value of the hop counter to zero. Further, it sends a special control message, referred to as a detach message, to the process at a distance of $D$ from it along the acknowledgment chain but in the reverse direction. The objective of a detach message is to instruct the intended recipient that it should break the link with its parent, become the "head" of the chain and report its status to the coordinator instead of reporting to its parent. (The details of how this happens are discussed in the previous paragraph.) The reason is that the overhead incurred on exchanging control messages with the coordinator, namely st_active, ack_st_active and st_passive, can now be amortized over enough number of processes so as not to affect the message complexity adversely. Note that a process may have multiple chains of acknowledgment messages emanating from it. As a result, there may be multiple processes that are at a distance of $D$ from it, all of which generate detach messages destined for it. This may increase the message complexity significantly. To that end, we propagate detach messages upward along an acknowledgment chain in a "modified" convergecast fashion. If a process has already sent a detach message to its parent since last becoming non-quiescent, then it ignores any subsequent detach message it receives from any of its other children (in the computation tree). Clearly, at most one detach message is sent in each non-quiescent interval. As a result, the total number of detach messages exchanged by the termination detection algorithm is upper-bounded by the total number of application messages exchanged by the underlying computation.

Example 1 Figure 1 illustrates the main idea behind our termination detection algorithm. Suppose process $p_{i}$, on receiving an engaging application message $m$, learns that the length of the acknowledgment chain has become at least $D$. Let the last $D+1$ processes along the chain be denoted by $p_{j}=p_{c_{0}}, p_{c_{1}}, \ldots, p_{c_{D}}=p_{i}$. As per our algorithm, $p_{i}$ generates a detach message and sends the message to its parent $p_{c_{D-1}}$. The detach message is propagated upward all the way to $p_{j}$, which is at a distance of $D$ hops from $p_{i}$. Process $p_{j}$, on receiving the detach message, sends an st_active message to the coordinator. The coordinator, on receiving the st_active message, sends an ack_st_active message to $p_{j}$. On receiving the ack_st_active message, $p_{j}$ sends an acknowledgment message to its parent, say process $p_{k}$, thereby breaking the chain. Numbers in the parentheses show the sequence in which various control messages are exchanged. It is possible that $p_{c_{D-1}}$ has another child, namely process $p_{l}$, which also sends a detach message to $p_{c_{D-1}}$ destined for $p_{j}$. On receiving the second detach message, $p_{c_{D-1}}$ simply ignores the message and does not forward it to its parent $p_{C_{D-2}}$.

Note that process $p_{i}$ is still attached to its parent $p_{c_{D-1}}$. Now, suppose the chain grows further by $D$ more processes and is now given by $p_{c_{0}}\left(=p_{j}\right), p_{c_{1}}, \ldots, p_{c_{D}}\left(=p_{i}\right), p_{c_{D+1}}$, $\ldots, p_{c_{2 D}}$. As per our algorithm, $p_{c_{2 D}}$ generates a detach message, which is propagated via processes $p_{c_{2 D-1}}, \ldots, p_{c_{D+1}}$ to $p_{i}$. Process $p_{i}$, on receiving the first detach message, breaks the link with its parent $p_{c_{D-1}}$, thereby reducing the length of the chain emanating from $p_{j}$.

Information description: The computation starts from the initially active process. As the computation exchanges application messages, a tree (sometimes referred to as computation tree) is induced on processes by engaging application messages. A tree grows whenever a process in the tree generates an engaging application message and shrinks whenever 


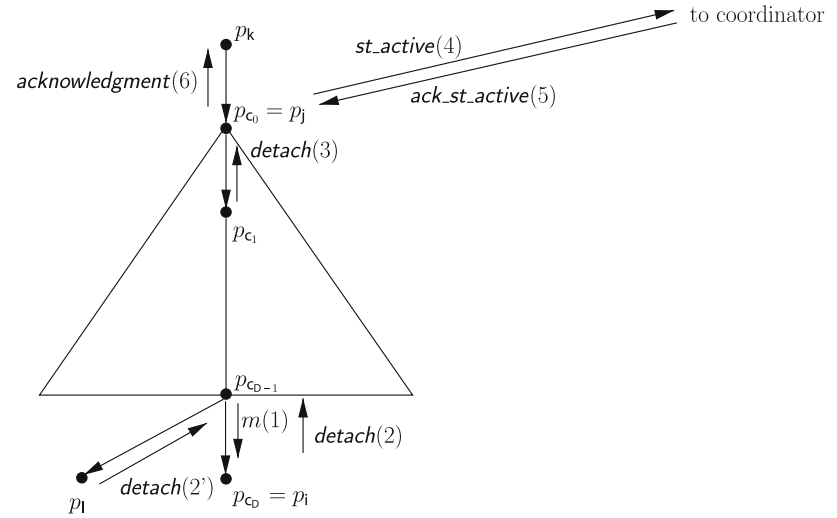

Fig. 1 An illustration of the termination detection algorithm

an engaging application message in the tree is acknowledged. Once the height of a subtree rooted at a process-for which the value of the hop counter is zero-becomes at least $D$, within $O(D)$ message hops, the process detaches itself from its parent and the subtree rooted at the process becomes a separate computation tree. A root process, which has detached itself from its parent, reports its status to the coordinator, and every other process reports its status to its parent in the tree. Whenever a tree becomes empty, its root process informs the coordinator about it. Once all trees have become empty, which happens once all application messages have been acknowledged, the coordinator announces termination. Our termination detection algorithm ensures that the coordinator announces termination if and only if there is no non-empty computation tree in the system.

Message-complexity: Our algorithm exchanges five different types of control messages, namely acknowledgment, detach, st_active, st_passive and ack_st_active. One acknowledgment message is exchanged for every application message. Also, a process sends at most one detach message for every engaging application message it receives. Therefore the total number of acknowledgment and detach messages is upper-bounded by $2 M$. The number of st_active messages generated by all processes combined is given by $O(M / D)$. This is because a process sends an st_active message only when it knows that there are at least $O(D)$ processes in its computation subtree. Each st_active message is sent on the BFS spanning tree and, therefore, may result in at most $D$ control messages being exchanged. Finally, the number of st_passive messages as well as the number of ack_st_active messages is equal to the number of st_active messages. Thus the message complexity of our algorithm is $O(M)$.

Detection-latency: Our algorithm ensures that whenever the length of a chain of pending acknowledgment messages grows beyond $2 D$, within $3 D+1$ message hops (consisting of detach, st_active and ack_st_active messages), the chain is reduced to a length smaller than $D$. Therefore the detection latency of our algorithm is $O(D)$.

\subsubsection{Generalizing to a non-diffusing computation}

Assume that two or more processes are active initially, that is, there are multiple initiators of the computation. Intuitively, the coordinator should announce termination only after every initiator has informed it that the computation triggered by it has terminated. The coordinator, however, does not know how many initiators of the computation are there. Therefore, every process, on becoming quiescent for the first time (including the case when it is quiescent to begin with), sends an initialize message to the coordinator. The coordinator announces termination only after it has received an initialize message from every process (and, of course, a matching st_passive message for every st_active message). The initialize messages are propagated to the coordinator in a convergecast fashion, thereby resulting in only $O(N)$ more messages.

\subsection{The algorithm}

A formal description of the termination detection algorithm TDA-SI for simultaneous initiation is given in Figs. 2, 3, and 4. Actions A0-A8 described in Figs. 2 and 3 capture the behavior of a process as part of the computation tree. Actions B1-B3 given in Fig. 4 describe the behavior of a process as part of the BFS spanning tree. The main function of a process as part of the spanning tree is to propagate messages, namely initialize, st_active, ack_st_active and st_passive, back and forth between the coordinator and its descendants in the spanning tree. For ease of exposition of the algorithm, we assume that whenever a process wants to send a control message to the coordinator (for instance, the initialize message), it sends that message to itself. The message is then handled either by action B1 or by action B2, and propagated upwards to the coordinator. Likewise, when a process receives an ack_st_active message from its parent in the spanning tree, it either propagates that message to one of its children in the spanning tree or sends the message to itself. In the latter case, the message is handled by action A6.

In the formal description our algorithm, whenever a process becomes non-quiescent, we classify it either as a root process or a non-root process. (The classification for process $p_{i}$ is captured using variable root $_{i}$.) The classification depends on how a process becomes non-quiescent. If a process is initially active, then it is classified as root. If a process becomes non-quiescent on receiving an application message with the counter value of $D-1$ (which is reset to zero on incrementing), then the process is classified as root as well. 


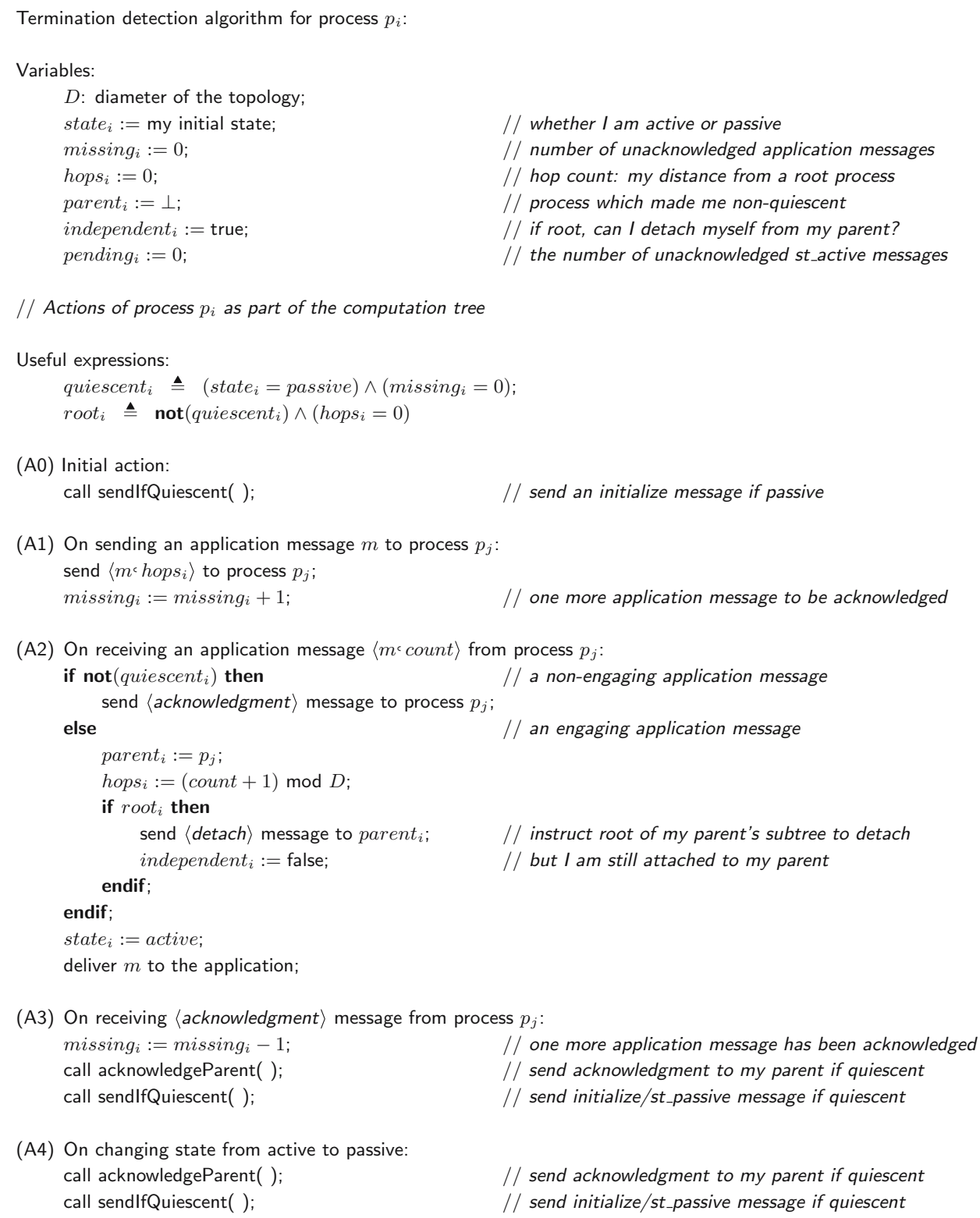

(A4) On changing state from active to passive: call acknowledgeParent( ); call sendlfQuiescent( );

Fig. 2 Termination detection algorithm TDA-SI for simultaneous initiation

In all other cases, a process is classified as non-root. Note that the same process may be classified as root and non-root at different times during its execution. However, the classification does not change during a single non-quiescent interval.

When a process becomes non-quiescent as a root, it does not immediately break its link with its parent, if it exists. It breaks the link only after receiving a detach message from one of its children in the computation tree. Receipt of a detach message implies that the computation subtree rooted at the process contains at least $D$ processes. As a result, the process can start reporting its status to the coordinator instead of its parent. The status of the link-whether it is intact or has been broken-is captured using the variable independent ${ }_{i}$.

We next prove that the termination detection algorithm TDA-SI described in Figs. 2, 3, and 4 is safe and live.

\subsection{Proof of correctness}

Many of our proofs involve induction on either the depth or the height of a vertex in a tree. Recall that the depth of a vertex $v$ in a tree, denoted by $\operatorname{depth}(v)$, is the length of the path from the root of the tree to $v$. Also, its height, denoted by height $(v)$, is the length of a longest path from $v$ to a leaf. 


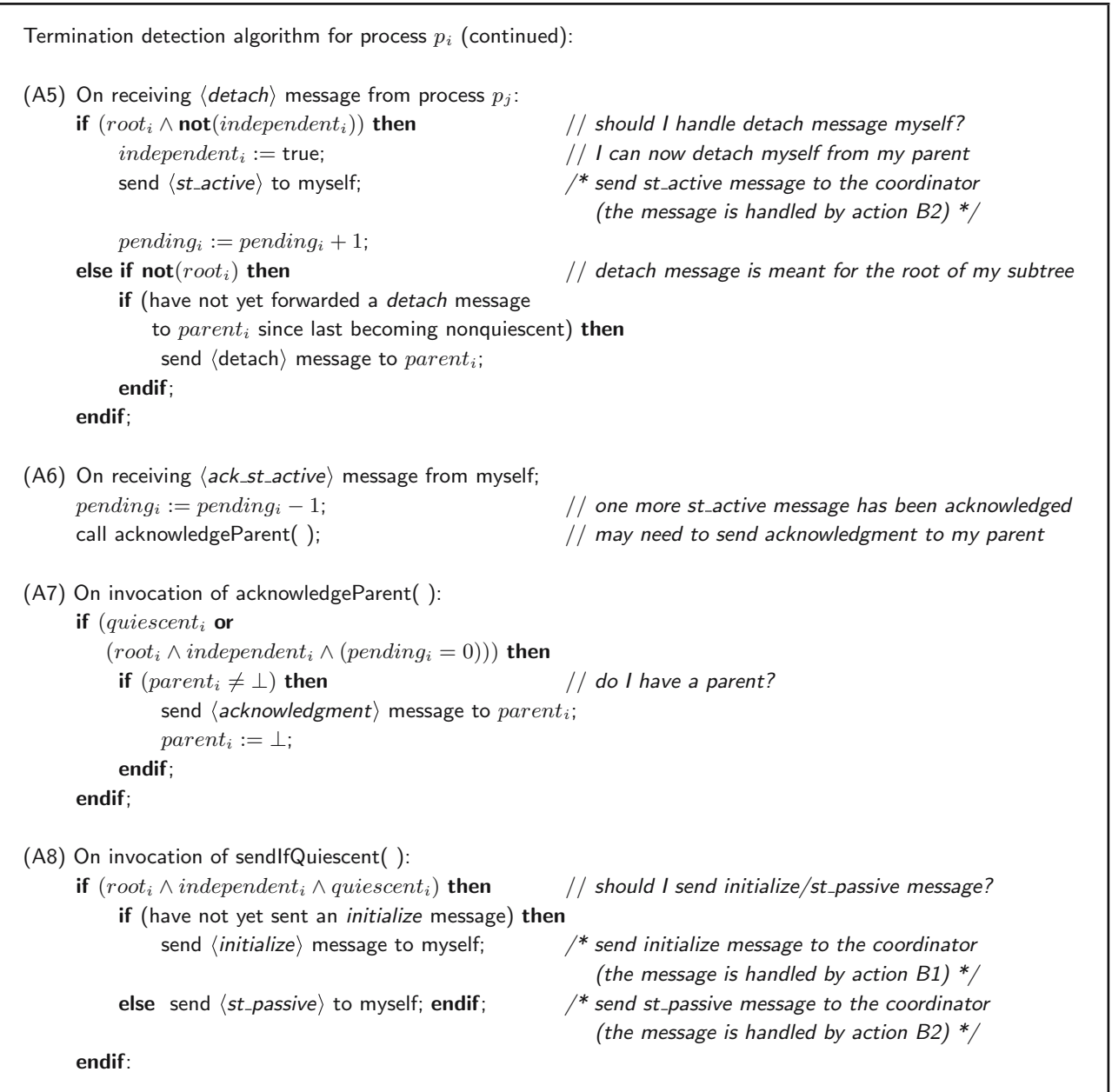

Fig. 3 Termination detection algorithm TDA-SI for simultaneous initiation (continued)

The two definitions can be easily generalized for vertex in a forest.

A process, on sending an st_active message to the coordinator, expects to receive an ack_st_active message eventually. Note that it is easy to route an st_active/st_passive message from a non-coordinator process to the coordinator. However, routing an ack_st_active message from the coordinator to the process that generated the corresponding st_active message is non-trivial. One approach to achieve this is by piggybacking the identity of the generating process on the st_active message which can then be used to appropriately route the corresponding ack_st_active message. This, however, increases the message overhead to $O(\log N)$. Moreover, with this approach, every process needs to know the set of descendants of each of its children in the static spanning tree. Instead, we employ the following mechanism. Every process on the BFS spanning tree propagates the $k$ th ack_st_active message to the sender of the $k$ th st_active message. This can be accomplished by maintaining a FIFO queue at each process that records the (immediate) sender of every st_active message that a process receives. Later, on receiving an ack_st_active message, the process uses the queue to forward the ack_st_active message to the appropriate process, which is either itself or one of its children. The next lemma can be proved by a simple induction on the depth of a process in the BFS spanning tree. The lemma states that if a process receives a matching ack_st_active message for its st_active message, then the coordinator "knows" that its subtree is "active"

Lemma 1 A process receives a matching ack_st_active message for its st_active message only after the st_active message has been received by the coordinator.

Proof The lemma can be proved by a simple induction on the depth of a process in the BFS spanning tree using the observation that every process sends the $k$ th ack_st_active message to the sender of the $k$ th st_active message.

We say that a process is quiescent if it is passive and has received an acknowledgment message for every application message it has sent so far. We partition the events on a 


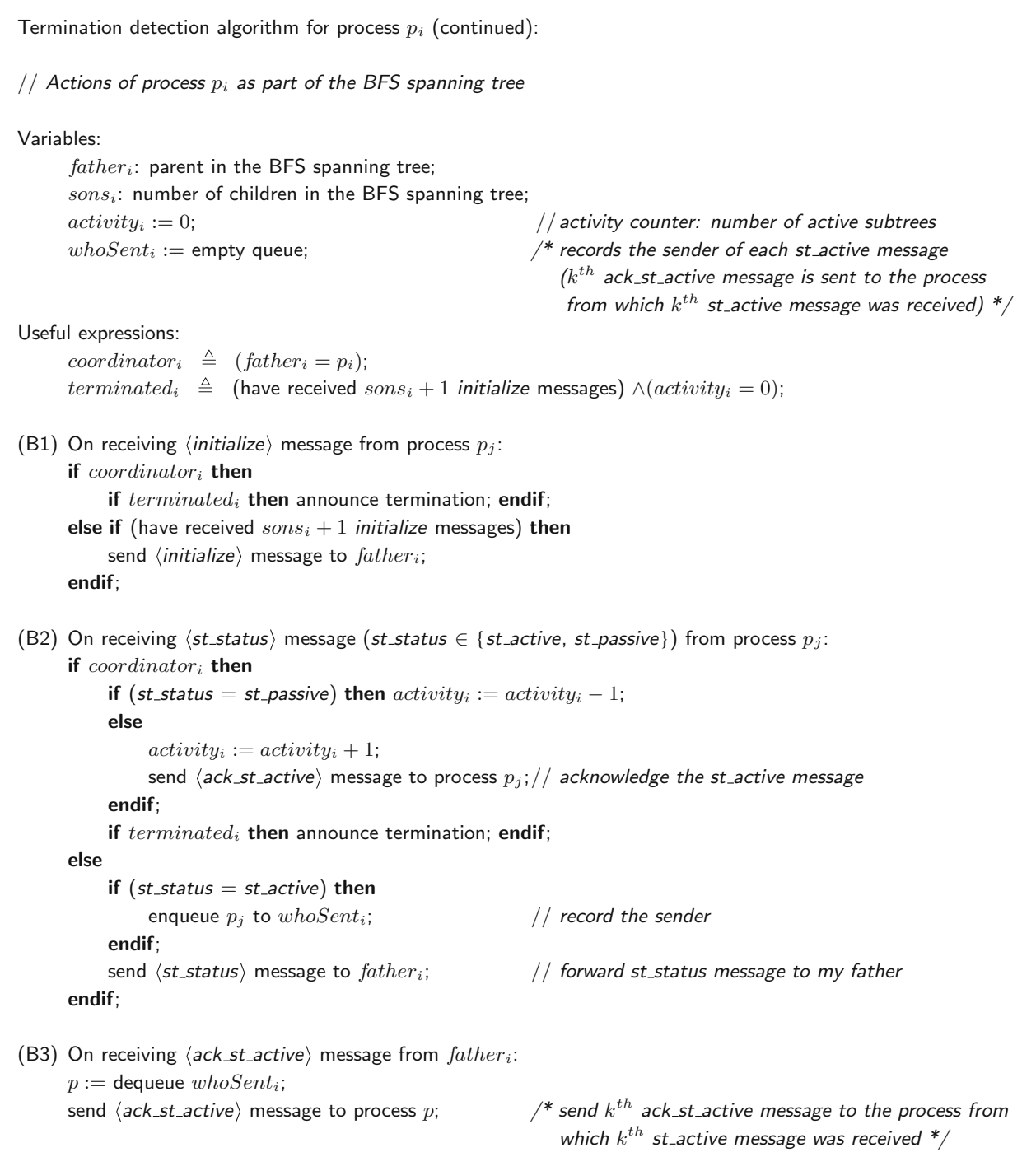

Fig. 4 Termination detection algorithm TDA-SI for simultaneous initiation (continued)

process into two categories: quiescent and nonquiescent. An event is said to be quiescent if the process becomes quiescent immediately after executing the event; otherwise it is nonquiescent. A maximal sequence of contiguous quiescent events on a process is called a quiescent interval. The notion of nonquiescent interval can be similarly defined. An interval is created as soon as its starting event is executed, and is completed once its last event is executed. An execution of a process can be viewed as an alternating sequence of quiescent and nonquiescent intervals.

We also partition the set of application messages into two categories: engaging and non-engaging. An application message is said to be engaging if its destination process, on receiving the message, changes its status from quiescent to nonquiescent; otherwise it is non-engaging.

Observe that the set of engaging application messages induces a forest (of trees) on the set of nonquiescent inter- vals. Specifically, given two nonquiescent intervals $x$ and $y$, there is an edge from $x$ to $y$ in the forest, denoted by $x \mapsto y$, if an engaging application message sent during $x$ is received during $y$. Let $N Q I$ denote the set of all nonquiescent intervals. It can be verified that $\langle N Q I, \mapsto\rangle$ is indeed a forest of trees. For an interval $x$, let $\operatorname{proc}(x)$ refer to the process on which events in $x$ are executed. The next lemma proves that if the computation terminates eventually, then the execution of every process ends with a quiescent interval.

Lemma 2 Assume that the underlying computation eventually terminates. Then, every nonquiescent process eventually becomes quiescent.

Proof Assume that the underlying computation has terminated. Therefore once a process becomes quiescent it stays quiescent. This implies that the set of nonquiescent intervals $N Q I$ is finite. The proof is by induction on the height of 
a nonquiescent interval in the forest $\langle N Q I, \mapsto\rangle$. A process acknowledges a non-engaging application message immediately. Thus it is sufficient to show that every engaging application message is eventually acknowledged. Consider a nonquiescent interval $x \in N Q I$ with $\operatorname{proc}(x)=p_{i}$.

Base case [height $(x)=0$ ]: In this case, all application messages sent in $x$ are non-engaging. Therefore process $p_{i}$ eventually becomes quiescent.

Induction step [height $(x)>0$ ]: Consider a nonquiescent interval $y$ with $x \mapsto y$. Clearly, height $(y)<$ height $(x)$. Therefore, using induction hypothesis, $\operatorname{proc}(y)$ eventually becomes quiescent. This, in turn, implies that $p_{i}$ eventually receives an acknowledgment message for the engaging application message it sends during $x$ to $\operatorname{proc}(y)$. Since $y$ is chosen arbitrarily, we can infer that $p_{i}$ eventually receives an acknowledgment message for every engaging application message it sends during $x$. Therefore $p_{i}$ eventually becomes quiescent.

From the algorithm, a process sends an initialize message when it becomes quiescent for the first time (including the case when it is quiescent to begin with). The following proposition can be easily verified:

Proposition 3 Assume that the underlying computation eventually terminates. Then, every process eventually sends an initialize message. Moreover, a process sends an initialize message only when it is quiescent for the first time.

It is important for the correctness of our algorithm that the coordinator receives st_active and st_passive messages in correct order. If channels are FIFO, then this can be achieved easily. If one or more channels are non-FIFO, then the algorithm has to be slightly modified. Details of the modifications required are described in Sect. 3.5. For now, assume that all channels are FIFO. We have,

Proposition 4 The st_active and st_passive messages sent by a process are received by the coordinator in the order in which they are sent.

The following lemma establishes that if the computation terminates then every process sends an equal number of st_active and st_passive messages in alternate order.

Lemma 5 Each process sends a possibly empty sequence of st_active and st_passive messages in an alternate fashion, starting with an st_active message. Furthermore, if the underlying computation eventually terminates, then every st_active message is eventually followed by an st_passive message.
Proof The execution of a process can be viewed as an alternating sequence of quiescent and nonquiescent intervals. If a process is initially passive, then the execution starts with a quiescent interval; otherwise it starts with a nonquiescent interval. Also, if the underlying computation eventually terminates, then, from Lemma 2 , the execution of every process ends with a quiescent interval.

From Proposition 3, every process sends an initialize message in the first quiescent interval. But the first interval for an initially active process is a nonquiescent interval. It can be verified that an initially active process does not send any st_active message in the first nonquiescent interval. This is because a process sends an st_active message only when it detaches itself from its parent in the computation tree (action A5).

Finally, it can be verified that after the first quiescent interval, if a process sends an st_active message in a nonquiescent interval, then it sends an st_passive message in the following quiescent interval.

We refer to the difference between the number of st_active and st_passive messages received by the coordinator as the activity counter. Using Proposition 4 and Lemma 5, it follows that:

Corollary 6 The activity counter at the coordinator always has a non-negative value. Moreover, immediately after processing an st_active message, the value of the activity counter is positive.

Also, from Lemma 5, it follows that:

Corollary 7 Assume that the underlying computation eventually terminates. Then, for every st_active message the coordinator receives, it eventually receives a matching st_passive message.

We are now ready to prove the correctness of our algorithm. First, we prove that our algorithm is live.

Theorem 8 (TDA-SI is live) Assume that the underlying computation eventually terminates. Then, the coordinator eventually announces termination.

Proof To establish the liveness property, it suffices to show that the following two conditions hold eventually. First, the coordinator receives all initialize messages it is waiting for. Second, the activity counter at the coordinator becomes zero permanently.

Note that initialize messages are propagated to the coordinator in a convergecast fashion. From Proposition 3, eventually every process sends an initialize message. It can be easily verified that every process on the BFS spanning tree will eventually send an initialize message to its parent in the spanning tree. As a result, the first condition holds eventually. 
For the second condition, assume that the underlying computation has terminated. Then, from Lemma 2 , every process eventually becomes quiescent and stays quiescent thereafter. This implies that every process sends only a finite number of st_active and st_passive messages. Therefore the coordinator also receives only a finite number of st_active and st_passive messages. Furthermore, from Corollary 7 , the coordinator receives an equal number of st_active and st_passive messages.

Finally, we prove that our algorithm is safe, that is, it never announces false termination.

Theorem 9 (TDA-SI is safe) The coordinator announces termination only after the underlying computation has terminated.

Proof Consider only those processes that become active at least once. Let announce denote the event on executing which the coordinator announces termination, and let $l q e_{i}$ denote the last quiescent event on process $p_{i}$ that happenedbefore announce. Such an event exists for every process. This is because the coordinator announces termination only after it has received all initialize messages it is waiting for. This, in turn, happens only after every process has sent an initialize message, which a process does only when it is quiescent.

Consider the snapshot $S$ of the computation consisting of all lqe events. Assume, on the contrary, that the computation has not terminated for $S$ and that some process becomes active after $S$. Let $N Q E$ denote the set of nonquiescent events executed in the future of $S$. Consider a minimal event mqe in NQE - minimal with respect to the happened-before relation. Formally,

$\langle\forall x: x \in N Q E: x \nrightarrow \rightarrow m q e\rangle$

Clearly, $m q e$ occurred on receiving an engaging application message, say $m$. Moreover, $m$ is a message sent from the past of $S$ to the future of $S$. Otherwise, it can be shown that $m q e$ is not a minimal event in $N Q E-$ a contradiction. Let $m$ be sent by process $p_{j}$ to process $p_{i}$. Also, let $\operatorname{snd}(m)$ and $r c v(m)$ correspond to the send and receive events of $m$, respectively. Then, $s n d(m) \rightarrow l q e_{j}$. This implies that $p_{j}$ becomes quiescent after sending $m$. Therefore it receives the acknowledgment message for $m$, denoted by $\operatorname{ack}(m)$, before executing $l q e_{j}$. This is depicted in Fig. 5a. There are two cases to consider:

Case 1 Process $p_{i}$ sends the acknowledgment message for $m$ on executing a quiescent event, say qe (see Fig. 5b). Clearly, the acknowledgment message creates a causal path from $q e$ to $l q e_{j}$. We have,

$$
\begin{aligned}
& \left(q e \text { is a quiescent event on } p_{i}\right) \wedge\left(l q e_{i} \rightarrow q e\right) \wedge \\
& \left(q e \rightarrow l q e_{j}\right) \wedge\left(l q e_{j} \rightarrow \text { announce }\right) \\
\Rightarrow \quad & \{\rightarrow \text { is transitive }\} \\
& \left(q e \text { is a quiescent event on } p_{i}\right) \wedge\left(l q e_{i} \rightarrow q e\right) \wedge \\
& (q e \rightarrow \text { announce })
\end{aligned}
$$

In other words, $q e$ is a quiescent event on $p_{i}$ that happenedbefore announce and is executed after $l q e_{i}$. This contradicts our choice of $l q e_{i}$.

Case 2 Process $p_{i}$ sends an acknowledgment message for $m$ before becoming quiescent. This happens only when $p_{i}$ receives an ack_st_active message for the st_active message it sends in the current non-quiescent interval (which starts with $m q e$ ). Let the receive event of the st_active message on the coordinator be denoted by $r c v_{c}$ (see Fig. 5c). Also, let the send event of $\operatorname{ack}(\mathrm{m})$ on process $p_{i}$ be denoted by $s n d_{i}$. Using Lemma $1, r c v_{c} \rightarrow s n d_{i}$. Therefore we have,

$$
\begin{aligned}
& \left(r c v_{c} \rightarrow s n d_{i}\right) \wedge\left(s n d_{i} \rightarrow l q e_{j}\right) \wedge\left(l q e_{j} \rightarrow \text { announce }\right) \\
\Rightarrow & \{\rightarrow \text { is transitive }\} \\
& r c v_{c} \rightarrow \text { announce }
\end{aligned}
$$

From Corollary 6, immediately after executing $r c v_{c}$, the value of the activity counter at the coordinator is greater than zero. For the coordinator to announce termination, its activity counter should be zero. This implies that the coordinator receives a matching st_passive message from $p_{i}$ later but before announcing termination. Clearly, $p_{i}$ sends this st_passive message only on executing some quiescent event after $m q e$. This again contradicts our choice of $l q e_{i}$.

We next prove that TDA-SI is both message-optimal and latency-optimal.

\subsection{Proof of optimality}

For a non-quiescent interval $x$ with $\operatorname{proc}(x)=p_{i}$, let hops $(x)$ denote the value of the variable hops $s_{i}$ during the interval $x$. From the algorithm (action A2),

$x \mapsto y \Rightarrow \operatorname{hops}(y)=(\operatorname{hops}(x)+1) \bmod D$

To prove the optimality of TDA-SI, the following proposition is useful.

Proposition 10 For a non-quiescent interval $x \in N Q I$ with hops $(x)=0$, if height $(x) \geqslant D$, then proc( $x)$ eventually receives a detach message during $x$ (that is, before the interval $x$ ends) and vice versa. 


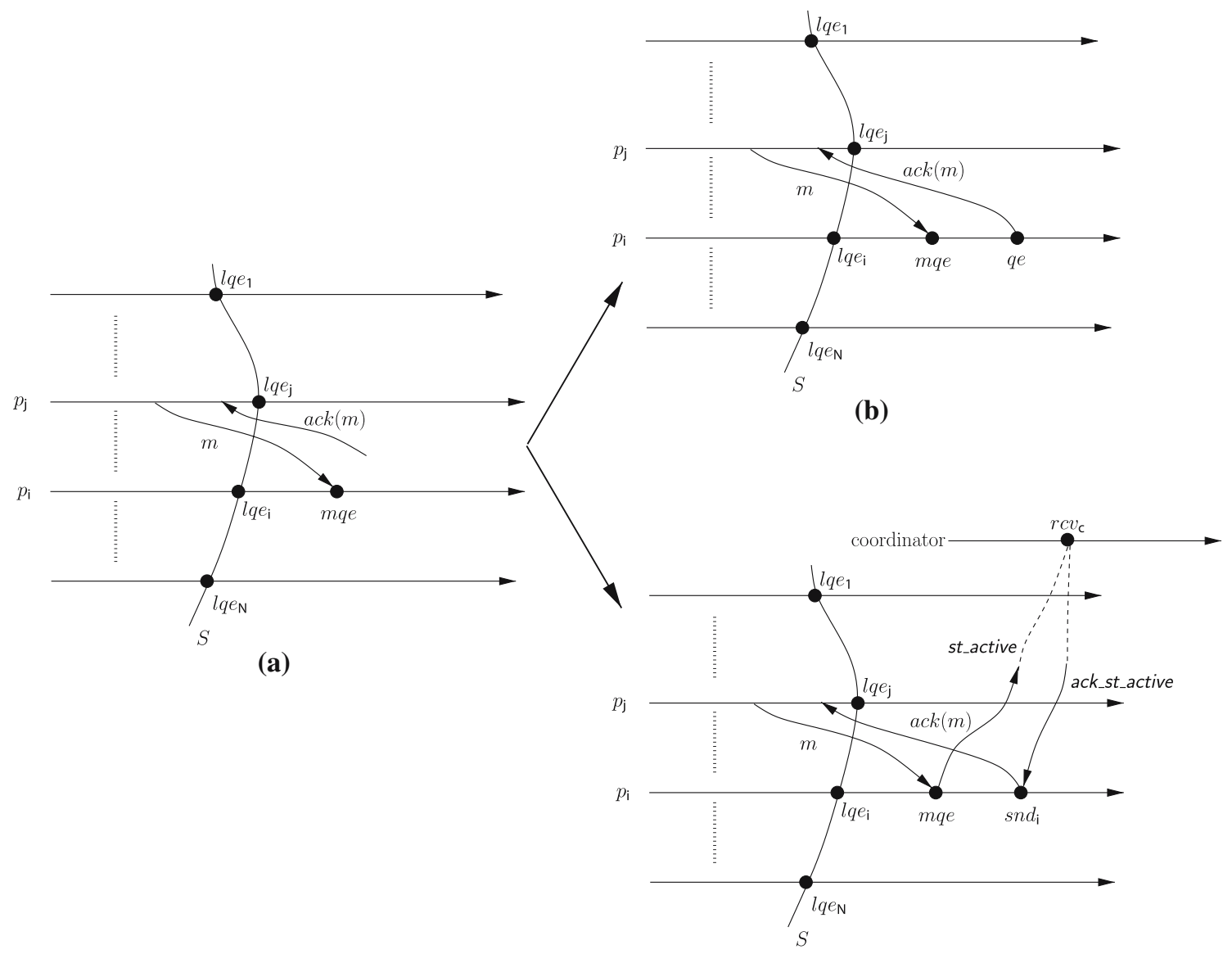

(c)

Fig. 5 Proving the safety of TDA-SI

The above proposition holds as long as the acknowledgment message for an engaging application message does not "overtake" any detach message sent earlier. Clearly, no "overtaking" occurs if all channels are FIFO. In case one or more channels are non-FIFO, the algorithm TDA-SI has to be modified slightly to ensure that Proposition 10 holds. Details of the modifications required are described in Sect. 3.5. We now show that our algorithm is message-optimal.

Theorem 11 (TDA-SI is message-optimal) Assume that the underlying computation eventually terminates. Then, the number of control messages exchanged by the algorithm is given by $\Theta(M+N)$, where $N$ is the number of processes in the system and $M$ is the number of application messages exchanged by the underlying computation.

Proof Our algorithm exchanges six different types of control messages, namely acknowledgment, detach, initialize, st_active, st_passive and ack_st_active. We now bound each of the six types of control messages.

The number of acknowledgment messages is same as the number of application messages $M$. A process sends at most one detach message per engaging application message.
Therefore the total number of detach messages is upperbounded by $M$. Every process sends at most one initialize message. Further, initialize messages are propagated to the coordinator in a convergecast fashion. Hence the total number of initialize messages exchanged by processes is given by $O(N)$.

Every st_active and st_passive message has to be propagated to the coordinator along the BFS spanning tree and, therefore, may cause up to $D$ additional (or derivative) control messages to be exchanged. Likewise, an ack_st_active message may also cause up to $D$ additional control messages to be exchanged. From Lemma 5, every process generates an equal number of st_active and st_passive messages. Moreover, the number of ack_st_active messages a process receives is equal to the number of st_active messages it sends. Thus it is sufficient to show that the total number of st_active messages generated by all processes combined is bounded by $M / D$.

Observe that a process sends an st_active message only when it is non-quiescent and, moreover, it sends at most one st_active message per non-quiescent interval (action A5). We, therefore, bound the number of non-quiescent inter- 
vals in which an st_active message is sent. Let $I \subseteq N Q I$ denote the set of those non-quiescent intervals during which an st_active message is sent. Also, observe that a process sends an st_active message during a non-quiescent interval only if the interval is created on receiving an engaging application message (action A5). In other words, if a process is non-quiescent to begin with, it does not send any st_active message during the (initial non-quiescent) interval. Let $N I \subseteq N Q I$ denote the set of non-quiescent intervals created on receiving an engaging application message. We have,

$I \subseteq N I \subseteq N Q I$ and $|N I| \leqslant M$

Consider a non-quiescent interval $x \in N Q I$. From the algorithm (action A5),

$x \in I \Rightarrow \operatorname{hops}(x)=0$

We define childset ( $x$ ) as the set of those non-quiescent intervals which are at a distance of at most $D-1$ message hops from $x$ in the forest $\langle N Q I, \mapsto\rangle$, and refer to it as the childset of $x$. Clearly, childset $(x) \subseteq N I$. Note that a process sends an st_active message during a non-quiescent interval only after it has received at least one detach message. Thus, from Proposition 10, (3) and the definition of I, height (x) is at least $D$ which implies that:

$x \in I \Rightarrow|\operatorname{childset}(x)| \geqslant D$

Since $\langle N Q I, \mapsto\rangle$ is a forest, from (3), (1) and the definition of childset,

$(\{x, y\} \subseteq I) \wedge(x \neq y) \Rightarrow$ childset $(x) \cap \operatorname{child} \operatorname{set}(y)=\emptyset$

We have,

$$
\begin{aligned}
& \left(\bigcup_{x \in I} \text { childset }(x)\right) \subseteq N I \\
\Rightarrow \quad & \{\text { using }(5)\} \\
& \left(\sum_{x \in I}|\operatorname{childset}(x)|\right) \leqslant|N I| \\
\Rightarrow & \{\text { using }(4) \text { and }(2)\} \\
& D \times|I| \leqslant\left(\sum_{x \in I}|\operatorname{childset}(x)|\right) \leqslant M \\
\Rightarrow & \{\text { algebra }\} \\
& |I| \leqslant M / D
\end{aligned}
$$

This establishes that TDA-SI is message-optimal.

We now show that our algorithm is latency-optimal. The next lemma states that once the underlying computation has terminated, no process stays non-quiescent for a "long" time.
Lemma 12 Once the underlying computation terminates, every process becomes quiescent within $O(D)$ message hops, where $D$ is the diameter of the communication topology.

Proof Assume that the underlying computation has terminated. Consider two processes $p_{i}$ and $p_{j}$ that are still nonquiescent just after the computation terminates. We say that $p_{i}$ is waiting on $p_{j}$ if $p_{i}$ has sent an engaging application message to $p_{j}$ but $p_{j}$ has not yet sent an acknowledgment for that message. Now, consider any chain formed using "waiting on" relationships that starts from process $p_{i}$ and whose length is at least $2 D$. Clearly, the chain consists of a process $p_{k}$ such that $p_{k}$ is at a distance of at most $D$ from $p_{i}$ in the chain and hops $k=0$. From the algorithm, $p_{k}$ receives a detach message within $D$ message hops of termination. After receiving the detach message, $p_{k}$ sends an st_active message to the coordinator for which it receives a matching ack_st_active message within $2 D$ message hops. After receiving the ack_st_active message, $p_{k}$ sends an acknowledgment message to its parent, if it has not already done so, causing the chain to break and become shorter. In other words, within $3 D+1$ message hops of termination, all chains of "waiting on" relationships are reduced to length smaller than $D$. Clearly, once that happens, all processes become quiescent within $D$ message hops.

Finally, we have,

Theorem 13 (TDA-SI is latency-optimal) Once the underlying computation terminates, the coordinator announces termination within $O(D)$ message hops.

Proof From Lemma 12, every process becomes quiescent within $O(D)$ message hops after the computation has terminated and stay quiescent thereafter. Therefore all initialize, st_active and st_passive messages are generated within $O(D)$ message hops of termination and no more messages are generated after that. Since the coordinator is at most $D$ message hops away from any process, the coordinator receives all initialize, st_active and st_passive messages within $O(D)$ message hops of termination soon after which it announces termination (action B2).

In the next section, we discuss modifications to our termination detection algorithm required in case one or more channels are non-FIFO.

\subsection{Dealing with non-FIFO channels}

To prove the correctness and optimality of the algorithm TDA-SI, we make the assumption that all channels are FIFO in two places. First, to ensure that the coordinator receives st_active and st_passive messages in the order in which they are sent (Proposition 4). Second, to ensure that the acknowledgment message for an engaging application messages does not "overtake" any detach message sent earlier 
(Proposition 10). In case one or more channels are non-FIFO, the following modifications to the algorithm can be used to ensure that both propositions still hold.

Ensuring that the coordinator receives st_active and st_passive messages in order: For convenience, we use st_status message to refer to an st_active message as well as an st_passive message, when it is not necessary to distinguish between the two. In the modified algorithm, the coordinator acknowledges all st_status messages, that is, both st_active and st_passive messages. Further, a process does not send the next st_status message until it has received an acknowledgment for its previous st_status message. This can be accomplished by maintaining a FIFO queue at each process. When a new st_status message is generated by a process, the message is buffered until the process has sent all previous st_status messages and, moreover, has received acknowledgments for all of them. It can be verified that the above two modifications do not affect the correctness and message-optimality of our algorithm. However, they may increase the detection latency. Specifically, it is possible that when the underlying computation terminates the queue still contains a large number of st_status messages. To prevent the queue from becoming too long, we can proceed as follows. A process, on generating an st_active message, checks to see if the queue contains an st_status message. If the queue is non-empty, then the process simply discards the (new) st_active message and also deletes the last st_status message, which will be an st_passive message, from the queue. Intuitively, the "new" st_active message "cancels" the "old" st_passive message. A similar optimization can be performed when an st_passive message is generated. This ensures that the queue never contains more than one pending st_status message.

Ensuring that the acknowledgment message for an engaging application message does not overtake any detach message sent earlier: In the modified algorithm, every detach message is acknowledged. Specifically, a process, after sending a detach message to its parent, waits until it has received an acknowledgment (for the detach message) from its parent before sending the acknowledgment message for the engaging application message. The notion of quiescence is now redefined as: a process is quiescent if it is passive, has received an acknowledgment message for every application message it has sent so far, and has received an acknowledgment for every detach message it has sent so far. It can be verified that the aforementioned modifications do not affect the correctness and optimality of our algorithm.

\subsection{Computing detection latency when $N / D$ is large}

In the analysis of the detection latency in Sect. 3.4, we assume that message processing time is negligible and can be ignored.
Message processing time can no longer be ignored if, for instance, the number of messages that a process has to handle in the worst-case, before termination can be announced, grows linearly with the number of processes. In that case, the detection delay due to message processing overhead will grow linearly with the number of processes in the system. On the other hand, the detection delay due to message transmission overhead will grow linearly with the diameter of the communication topology. As a result, when $N / D$ becomes sufficiently large, message processing overhead will start dominating message transmission overhead. In this section, we present an analysis of the detection latency by explicitly considering message processing time [20,21]. We assume that messages are processed in the order in which they are received along a channel. To avoid confusion between the terms "process" and "processing", we sometimes use the term "node" instead of "process".

When a message arrives along an incoming channel at a node, its processing may be delayed because of two reasons. First, there are a large number of other messages already waiting to be processed at the node. Second, messages continue to arrive at other incoming channels and the node chooses to process them first, thereby "ignoring" some incoming channel for a long time. Although both scenarios are possible in an asynchronous distributed system, they do not occur often in practice. Typically, the system maintains a limited amount of buffer space for each channel to store messages that have not been processed by the application yet. Likewise, the probability that some incoming channel is not serviced by the application for a long time even though it has a message waiting to be received is very small. Therefore we make the following two realistic assumptions. The first assumption is concerned with the number of messages that may be waiting to be processed at a node at any given time.

Assumption 1 (bounded buffer) At any give time, the number of messages waiting to be processed along an incoming channel at a node is bounded by $B$, where $B$ is some constant.

The second assumption is concerned with the fairness of processing messages at a node.

Assumption 2 (bounded wait) Assume that a message received along an incoming channel $c$ at a node is waiting to be processed. Then, the node may process at most $W$ messages along each of its other incoming channels before processing any message received along the channel $c$, where $W$ is some constant.

Assume that the computation has terminated. Table 2 describes the states through which the system passes before termination is announced. Note that each of the property defined in the table is stable in the sense that the property continues to hold once it becomes true. As we are interested in computing the detection latency in the worst-case, we assume that 
Table 2 States through which a system passes once the computation terminates but before termination is announced

Property satisfied by the system state

Earliest time at which the property becomes true

$P_{0}:$ the computation has terminated

$t_{0}$

$P_{1}$ : all nodes have received and processed acknowledgment messages for their non-engaging application messages $t_{1}$

$P_{2}$ : all root nodes with large computation subtrees have received and processed at least one detach message $\quad t_{2}$

$P_{3}$ : all root nodes with large computation subtrees have broken their links with their parents $t_{3}$

$P_{4}$ : all processes have become quiescent $\quad t_{4}$

$P_{5}:$ the coordinator has announced termination

$t_{5}$

$t_{i+1}>t_{i}$ for each $i=0, \ldots, 4$. We bound the length of the interval $t_{i+1}-t_{i}$ for each $i=0, \ldots, 4$.

Let $\tau_{p}$ and $\tau_{c}$ denote the worst-case message processing and transmission time, respectively. Also, for a process $p_{i}$, let $\delta_{i}$ denote the degree of process $p_{i}$ in the communication topology. We first bound $t_{1}-t_{0}$. Note that acknowledgment messages for non-engaging application messages are sent before the computation terminates. These messages take at most $\tau_{c}$ time to arrive at their respective destination processes. Once an acknowledgment message arrives at a node, say $p_{i}$, it is processed within $O\left(B W \delta_{i} \tau_{p}\right)$ time. Let $\Delta$ denote the maximum degree of a node in the communication topology. Since $\delta_{i} \leqslant \Delta$, we have:

$t_{1}-t_{0}=O\left(\tau_{c}+B W \Delta \tau_{p}\right)$

We now bound $t_{2}-t_{1}$. We define the fan-out of a communication topology as the minimum of $\Delta \cdot D$ and $2 N$ and denote it by $F$. Formally,

\section{$F \triangleq \min \{\Delta \cdot D, 2 N\}$}

Intuitively, we use fan-out to capture the worst-case delay experienced by a message traveling a distance of at most $D$ hops along any tree of the topology. For a process $p_{i}$, let $s_{i}$ denote the degree of $p_{i}$ in the BFS spanning tree. Note that, at time $t_{1}$, all unacknowledged application messages are engaging in nature. Let $c_{i}$ denote the degree of $p_{i}$ in the computation tree (more precisely, forest) induced by unacknowledged application messages at time $t_{1}$. Note that both $s_{i}$ and $c_{i}$ are at most $\Delta$. Let $Q$ denote some subset of nodes. When $Q$ contains at most $D$ nodes, the following inequalities can be easily verified:

$$
\sum_{p_{i} \in Q \text { and }|Q| \leqslant D} s_{i} \leqslant F \text { and } \sum_{p_{i} \in Q \text { and }|Q| \leqslant D} c_{i} \leqslant F
$$

After $t_{1}$, a process $p_{i}$ can receive a message along an incoming channel only if the channel is a part of either the BFS spanning tree or the computation tree. In other words, after $t_{1}$, $p_{i}$ can receive messages along at most $s_{i}+c_{i}$ incoming channels. Further, at $t_{1}$, all messages received along the remaining incoming channels have already been processed by $p_{i}$. To bound $t_{2}-t_{1}$, we proceed as follows. Observe that all detach messages are generated before the computation terminates. A detach message has to travel $D$ hops along a chain of unacknowledged application messages in the reverse direction. Let the chain of processes through which a detach message passes be given by $p_{i_{0}}, p_{i_{1}}, \ldots, p_{i_{D}}$, where $p_{i_{0}}$ generates a detach message intended for $p_{c_{D}}$. When a detach message arrives at a process $p_{i_{j}}$, where $0<j \leqslant D$, it is processed within $O\left(B W\left(s_{i_{j}}+c_{i_{j}}\right) \tau_{p}\right)$ time. As a result, once $p_{i_{0}}$ generates a detach message, $p_{i_{D}}$ receives and processes a detach messages within $\sum_{j=1}^{D} O\left(\tau_{c}+B W\left(s_{i_{j}}+c_{i_{j}}\right) \tau_{p}\right)$ time. Therefore, using (7), we have:

$$
\begin{aligned}
t_{2}-t_{1} & =\sum_{j=1}^{D} O\left(\tau_{c}+B W\left(s_{i_{j}}+c_{i_{j}}\right) \tau_{p}\right) \\
& =O\left(D \tau_{c}+B W \tau_{p} \sum_{j=1}^{D}\left(s_{i_{j}}+c_{i_{j}}\right)\right) \\
& =O\left(D \tau_{c}+B W F \tau_{p}\right)
\end{aligned}
$$

Using a similar argument, we can show that $t_{3}-t_{2}, t_{4}-t_{3}$ and $t_{5}-t_{4}$ are all bounded by $O\left(D \tau_{c}+B W F \tau_{p}\right)$. The main idea is that once a property $P_{i}$ holds, where $2 \leqslant i<$ 5 , property $P_{i+1}$ holds after some messages have traveled at most $O(D)$ hops. Further, the number of such messages is small (specifically, $O(1)$ ). Combining the bounds on all intervals, it follows that $t_{5}-t_{0}$ is bounded by $O\left(D \tau_{c}+\right.$ $\left.B W F \tau_{p}\right)$.

When message processing is considered explicitly, the lower bound on detection latency of a termination detection algorithm for a communication topology is no longer given by $\Omega(D)$. But rather it is given by the minimum amount of time it takes to perform a broadcast (or a convergecast) on that topology [21]. Therefore our termination detection algorithm is latency-optimal whenever the lower bound on the time-complexity of a doing a broadcast on a communication topology is $\Omega(F)$. Some examples of such topologies include chain, ring, star and a grid. 


\section{An optimal algorithm for delayed initiation}

If the underlying computation is message-intensive, then it is desirable not to initiate the termination detection algorithm along with the computation. It is preferable, instead, to initiate it later, when the underlying computation is "close" to termination. This is because, in the latter case, the (worstcase) message-complexity of the termination detection algorithm would depend on the number of application messages exchanged by the computation after the termination detection algorithm has commenced. As a result, with delayed initiation, the termination detection algorithm generally exchanges fewer number of control messages than with simultaneous initiation.

To correctly detect termination with delayed initiation, we use the scheme proposed in [4]. The main idea is to distinguish between application messages sent by a process before it started termination detection and messages sent by it after it started termination detection. Clearly, the former messages should not be "tracked" by the termination detection algorithm and the latter messages should be "tracked" by the termination detection algorithm. Note that delayed initiation is not possible unless all channels are FIFO. This is because if one or more channels are non-FIFO then an application message may be delayed arbitrarily on a channel, no process would be aware of its existence, and this message may arrive at the destination after termination has been announced. Therefore we assume that all channels are FIFO. We also assume that each process knows all its neighboring processes (that is, outgoing channels).

In order to distinguish between the two kinds of application messages, we use a marker message. Specifically, as soon as a process starts the termination detection algorithm, it sends a marker message along all its outgoing channels. Therefore, when a process receives a marker message along an incoming channel, it knows that any application message received along that channel from now on has to be acknowledged as per the termination detection algorithm. On the other hand, if a process receives an application message on an incoming channel along which it has not yet received a marker message, then that message should not be acknowledged and should be simply delivered to the application. Intuitively, a marker message sent along a channel "flushes" any in-transit application messages on that channel. For ease of exposition, we assume that initially all incoming channels are uncolored. Further, a process, on receiving a marker message along an incoming channel, colors the channel along which it has received the marker message.

To initiate the termination detection algorithm, the coordinator sends a marker message to itself. When a process receives a marker message, as explained before, it colors the incoming channel along which the marker message is received. Additionally, if it is the first marker message to be received, the process starts executing the termination detection algorithm and also sends a marker message along all its outgoing channels. Note that the coordinator should not announce termination at least until every process has received a marker message along all its incoming channels and therefore has colored all its incoming channels. Otherwise, some uncolored channel may contain an application message that neither the sender nor the receiver is aware of and the message may arrive after the termination is announced. This will violate the safety of the detection algorithm. To that end, we redefine the notion of quiescence as follows: a process is quiescent if it is passive, has received an acknowledgment message for every application message it has sent since it started executing the termination detection algorithm, and all its incoming channels have been colored. A formal description of the termination detection algorithm TDA-DI for delayed initiation is given in Fig. 6.

Once the coordinator starts the termination detection algorithm, all incoming channels are colored within $O(D)$ message hops. The following theorem can be proved in a similar manner as Lemma 2:

Lemma 14 Once the underlying computation terminates, all processes eventually become quiescent.

Moreover, using the definition of quiescence and the fact that all channels are FIFO, it follows that:

Lemma 15 If all processes are quiescent, then no channel contains an application message that was sent by a process before starting the termination detection algorithm.

From the above lemma, we can infer that:

Lemma 16 If all processes are quiescent, then the underlying computation has terminated.

Proof Assume that all processes are quiescent. Therefore every application message that was sent by a process after starting the termination detection algorithm has been acknowledged. This implies that no channel contains an application message that was sent by a process after starting the termination detection algorithm. Moreover, from Lemma 15, no channel contains an application message that was sent by a process before starting the termination detection algorithm. In other words, all channels are empty (of application messages). Moreover, since all processes are quiescent, they are passive as well.

Intuitively, TDA-DI announces termination once it detects that all processes have become quiescent, and vice versa. Therefore its liveness follows from Lemma 14 and its safety follows from Lemma 16.

The only additional messages exchanged by TDA-DI are marker messages. Therefore the message-complexity of TDA-DI is $O(\bar{M}+E)$, where $\bar{M}$ is number of application 


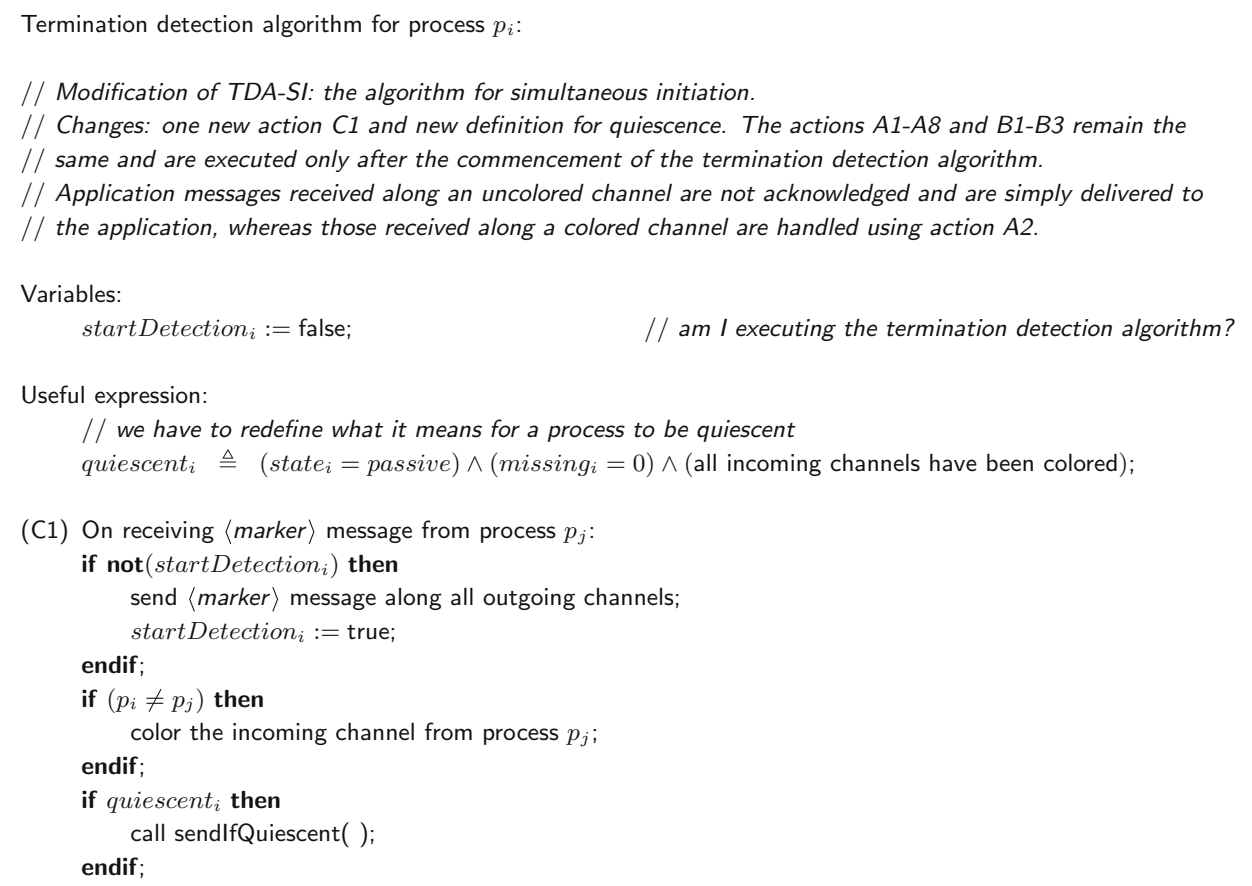

Fig. 6 Termination detection algorithm TDA-DI for delayed initiation

messages exchanged by the distributed computation after the termination detection algorithm has started and $E$ is the number of channels in the communication topology. Note that $\bar{M}$ may be as large as $M$ in the worst case. Therefore the worst-case message complexity of the termination detection algorithm with delayed initiation is actually more than that of the algorithm with simultaneous initiation. However, we expect the average message complexity to be much lower in the case of delayed initiation because much fewer application messages will need to be tracked on average.

Also, assuming that the termination detection algorithm is started before the underlying computation terminates, the detection latency of TDA-DI is $O(D)$. This is because, once the coordinator starts the termination detection algorithm, within $O(D)$ message hops, all processes start the termination detection algorithm and all incoming channels become colored. After this, similar to Lemma 12 and Theorem 13, it can be proved that all processes become quiescent and termination is detected within $O(D)$ message hops. Elsewhere, we show that any termination detection algorithm designed for simultaneous initiation can be transformed into a termination detection algorithm for delayed initiation with minimal impact on its performance [28].

\section{An optimal algorithm when application messages are multi-hop}

In this section, we describe modifications required to our algorithm to ensure optimality with respect to message- complexity and detection-latency when application messages can be exchanged between arbitrary processes. We describe the modifications assuming simultaneous initiation. The ideas in this section can be easily combined with the ideas in the previous section to maintain optimality with delayed initiation as well.

We assume that an application message sent by process $p_{i}$ to process $p_{j}$ travels along a shortest path from $p_{i}$ to $p_{j}$. We also assume that any acknowledgment or detach message that $p_{j}$ sends to $p_{i}$ travels along the path taken by the application message in reverse direction.

\subsection{Modifications to our algorithm}

When application messages can be exchanged between arbitrary processes (and not just neighboring processes), each link in an acknowledgment chain may consist of $\Theta(D)$ message hops in the worst-case. As a result, the length of the acknowledgment chain in terms of number of message hops may be as large as $\Theta\left(D^{2}\right)$. This means that the worstcase detection latency of our algorithm is $\Theta\left(D^{2}\right)$, which is clearly suboptimal.

To achieve latency-optimality, instead of incrementing the hop counter by one every time an application message is exchanged, we increment the counter by the number of hops in the path taken by the application message. Observe that, with this modification, the value of the hop counter can actually become more than $D$. The counter is reset to zero as soon as its value becomes greater than or equal to $D$. 
This ensures that the value of the counter never exceeds $2 D$, which, in turn, implies that the length of the acknowledgment chain in terms of number of message hops never exceeds $2 D$.

As before, when a process resets the hop counter, its behavior is similar to that of a root process in TDA-SI. Specifically, it generates a detach message that is propagated upwards to the closest root process in the computation tree, which is at a distance of at least $D$ message hops from it. Further, it maintains its link with its parent and reports its status to it until it receives a detach message from one of its children in the computation tree.

Clearly, our modifications ensure that the detection latency of the resulting algorithm is $O(D)$ in the worst-case. We now show that the modified algorithm has optimal messagecomplexity as well. We refer to the modified algorithm as TDA-SI-MH.

\subsection{Proof of message-optimality}

Note that, in TDA-SI-MH, a st_active or st_passive message generated by a process may not be amortized over at least $D$ application messages. This is because the acknowledgment chain is cut as soon as its length becomes at least $D$ in term of number of message hops and not in terms of number of application messages. In fact, in the worst case, the length of the acknowledgment chain may consist of only one application message (that travels a distance of $D$ message hops). As a result, it may appear that TDA-SI-MH is no longer message-optimal. We show that our algorithm is still message-optimal if we count a control message (such as an acknowledgment message or a detach message) that travels a distance of $d$ message hops as $d$ different control messages. This is also consistent with the way we count st_active, st_passive and ack_st_active messages in the analysis of TDA-SI-MH.

Let $H$ denote the average number of hops traveled by an application message. It is given by the ratio total number of hops traveled by all application messages total number of application messages

Note that $1 \leqslant H \leqslant D$. When application messages are only exchanged between neighboring processes, clearly, $H=1$. We show that the message complexity of the modified algorithm is $\Theta(M H+N)$, which we prove is optimal.

First, we show that the worst-case message complexity of an arbitrary termination detection algorithm $\mathcal{A}$ is given by $\Omega(M H+N)$, thereby proving the optimality of TDA-SI-MH. It suffices to show that the worst-case message complexity of any termination detection algorithm is $\Omega(M H)$ when the computation is diffusing. Note that one may be tempted to think that the lower bound trivially follows from Chandy and Misra's lower bound proof [6] by replacing each application message that travels a distance of $d$ hops with $d$ application messages, each of which travels a distance of one hop. However the transformation is not correct for the following reason: Suppose an application message from process $p_{i}$ to process $p_{j}$ travels via processes $p_{k_{1}}, p_{k_{2}}, \ldots, p_{k_{d-1}}$. Then, before the transformation, each process $p_{k_{l}}$, where $1 \leqslant l<d$, basically acts as a relay; it does not become active on receiving the application message. However, after the transformation, each process $p_{k_{l}}$, where $1 \leqslant l<d$, has to become active on receiving the corresponding application message to satisfy the rules of the computation. Another approach is to assume that an "intermediate" application message does not really spawn any activity in the system in the sense that a passive process on receiving such a message stays active for a very short while during which it simply forwards the message to the next process. However, Chandy and Misra's lower bound proof [6] assumes that each application message is capable of spawning independent activity in the system and therefore the proof does not carry over to the system obtained after the transformation.

Note that, if there exists a computation state reachable from the initial state after which the termination detection algorithm $\mathcal{A}$ exchanges an infinite number of control messages, then the lower bound trivially holds. Therefore assume that, after each computation state reachable from the initial state, the termination detection algorithm $\mathcal{A}$ exchanges only a finite number of control messages after which it does not exchange any control message until the computation executes an event. Our lower bound proof uses the following lemma.

Lemma 17 Consider two processes $p_{i}$ and $p_{j}$ that are at distance of $H$ hops from each other. Assume that the system is in a state in which only $p_{i}$ and $p_{j}$ are active, all other processes are passive and all channels are empty. Then there exist an execution $\sigma$ of the system (starting from the given state) and a process $p_{k} \in\left\{p_{i}, p_{j}\right\}$ such that (1) after $\sigma$ only $p_{k}$ is active, all other processes are passive and all channels are empty, (2) no application message is exchanged during $\sigma$, and (3) at least $\lceil H / 2\rceil$ control messages are exchanged by an arbitrary termination detection algorithm $\mathcal{A}$ during $\sigma$.

Proof We consider two executions of the computation from the given state: one in which both processes become passive and one in which only one of them becomes passive.

In the first execution of the computation, denoted by $\kappa_{1}$, both processes $p_{i}$ and $p_{j}$ become passive without generating any application message. Clearly, once that happens, the computation terminates. Consider an execution $\tau_{1}$ of the termination detection algorithm $\mathcal{A}$ from the state resulting after executing $\kappa_{1}$ such that (1) after $\tau_{1}$, no more control messages are exchanged, and (2) some process announces termination in $\tau_{1}$. Such an execution exists because, by assumption, the termination detection algorithm exchanges only a finite number of messages in any computation state and, moreover, it is live. Let $p_{t}$ be a process that announces termination in 
$\tau_{1}$. Process $p_{t}$ can announce termination only after "learning" that both $p_{i}$ and $p_{j}$ have become passive. Otherwise, it can be shown that the termination detection algorithm is not safe. Note that, in an asynchronous distributed system, the knowledge that some process has become passive can only be acquired through a (possibly empty) causal chain of messages [6]. Let $K$ denote the set of processes that "learn" during the execution $\tau_{1}$ of the algorithm that both $p_{i}$ and $p_{j}$ have become passive. The set $K$ is non-empty because $p_{t} \in K$. For a process $p_{x} \in K$, let first $\left(p_{x}\right)$ denote the earliest event on $p_{x}$ when $p_{x}$ acquired that knowledge during $\tau_{1}$. Consider a process $p_{\min } \in K$ such that first $\left(p_{\min }\right)$ is a minimal event, with respect to $\rightarrow$, among all events in $\left\{\right.$ first $\left.\left(p_{x}\right) \mid p_{x} \in K\right\}$. Note that $p_{\min }$ is at a distance of at least $\lceil H / 2\rceil$ hops from either $p_{i}$ or $p_{j}$. Without loss of generality, assume that $p_{\min }$ is at a distance of at least $\lceil H / 2\rceil$ hops from $p_{i}$. For $p_{\min }$ to "learn" that $p_{i}$ has become passive, there should be causal chain of messages starting from when $p_{i}$ becomes passive and ending at first $\left(p_{\min }\right)$ such that all messages in the chain are sent during $\tau_{1}$. Let $C$ denote the set of processes through which this chain passes. ( $C$ includes $p_{i}$ but does not include $p_{\min }$.) Clearly, $C$ contains at least $\lceil H / 2\rceil$ processes. Moreover, from the way $p_{\min }$ is chosen, each process in $C$ sends its first control message during $\tau_{1}$ without knowing that the other process $p_{j}$ has become passive.

Now, consider the second execution of the computation, denoted by $\kappa_{2}$, in which only process $p_{i}$ becomes passive but $p_{j}$ remains active. Processes in $C$ clearly cannot distinguish between executions $\kappa_{1}$ and $\kappa_{2}$ of the computation when they send their first control message during $\tau_{1}$. Therefore there exists an execution $\tau_{2}$ of the termination detection algorithm from the state resulting after $\kappa_{2}$ such that (1) at least $\lceil H / 2\rceil$ processes send a control message during $\tau_{2}$, and (2) no control message is sent after $\tau_{2}$ until the computation executes an event. The required execution $\sigma$ of the system is given by $\kappa_{2}$ followed by $\tau_{2}$.

We are now ready to prove the lower bound on message complexity of a termination detection algorithm. Our proof is based on the proof of lower bound on message complexity of a termination detection algorithm given in Tel [31] for the case when $H=1$.

Theorem 18 (lower bound on message-complexity) Consider a diffusing computation and assume that the computation eventually terminates. Then, the worst-case messagecomplexity of any termination detection algorithm is given by $\Theta(M H)$, where $M$ is the number of application messages exchanged by the underlying computation and $H$ is the average number of hops traveled by the application messages.

Proof The proof is constructive by nature. We construct a system execution in steps. In each step, the underlying computation exchanges one application message that travels a distance of $H$ hops because of which the termination detection algorithm is forced to exchange $\Omega(H)$ control messages.

Consider two processes $p_{i}$ and $p_{j}$ that are at a distance of $H$ hops from each other. Assume that the system is in a state $X$ in which only one process, say $p_{i}$, is active, all other processes are passive and all channels are empty. (This state may be the initial state of the system.) Now, suppose $p_{i}$ sends an application message to $p_{j}$ which makes $p_{j}$ active. Since the termination detection algorithm eventually stops exchanging control messages, the system eventually reaches a state $Y$ - via an execution $\sigma_{1}$-in which both $p_{i}$ and $p_{j}$ are active, all other processes are passive and all channels are empty. We can now apply Lemma 17 to system state $Y$. Thus there exists an execution $\sigma_{2}$ of the system that takes the system to a state $Z$ such that (1) in $Z$ only one process $p_{k} \in\left\{p_{i}, p_{j}\right\}$ is active, all other processes are passive and all channels are empty, (2) no application message is exchanged during $\sigma_{2}$ and (3) at least $\Omega(H)$ control messages are exchanged during $\sigma_{2}$.

Combining the two executions, we can conclude that there exists an execution $\sigma$ of the system, which is given by $\sigma_{1}$ followed by $\sigma_{2}$, such that (1) exactly one application message is exchanged during $\sigma$, (2) the application message travels a distance of $H$ hops, (3) at least $\Omega(H)$ control messages are exchanged during $\sigma$, and (4) the system state after $\sigma$ is isomorphic to the system state before $\sigma$.

The last property implies that the above-described construction can be repeated ad infinitum, thereby proving the lower bound.

Next, we show that TDA-SI-MH has optimal messagecomplexity.

Theorem 19 (TDA-SI-MH is message-optimal) Assume that the underlying computation eventually terminates. Then, the number of control messages exchanged by the modified algorithm is given by $\Theta(M H+N)$, where $N$ is the number of processes in the system, $M$ is the number of application messages exchanged by the underlying computation and $H$ is the average number of hops traveled by the application messages.

Proof The structure of the proof is quite similar to the structure of the proof for Theorem 11. We present it for the sake of completeness.

Our algorithm exchanges six different types of control messages, namely acknowledgment, detach, initialize, st_active, st_passive and ack_st_active. We now bound each of the six types of control messages.

Clearly, the number of acknowledgment messages is equal to the total number of hops traveled by all application messages collectively, which is given by $M H$. A process sends at most one detach message per engaging application message. Therefore the number of detach messages is upper-bounded by $M H$. Every process sends at most one initialize message. Further, initialize messages are propagated 
to the coordinator in a convergecast fashion. Hence the total number of initialize messages exchanged is given by $O(N)$.

Every st_active and st_passive message has to be propagated to the coordinator along the BFS spanning tree and, therefore, may have to travel a distance of up to $D$ message hops. Likewise, an ack_st_active message may have to travel a distance of up to $D$ message hops. From Lemma 5, every process sends an equal number of st_active and st_passive messages. Moreover, the number of ack_st_active messages a process receives is equal to the number of st_active messages it sends. Thus it is sufficient to show that the total number of st_active messages generated by all processes combined is bounded by $M H / D$. This in turn would imply that the total number of st_active, st_passive and ack_st_active messages exchanged is $O(M H)$.

Observe that a process sends an st_active message only when it is non-quiescent and, moreover, it sends at most one st_active message per non-quiescent interval. We, therefore, bound the number of non-quiescent intervals in which an st_active message is sent. Let $I \subseteq N Q I$ denote the set of those non-quiescent intervals during which an st_active message is sent. For two non-quiescent intervals $x$ and $y$ with $x \mapsto y$, the distance between $x$ and $y$ is given by the number of hops traveled by the engaging application message that created $y$ (and was sent during $x$ ). Our proof uses the notion of childset of a non-quiescent interval, which was defined in the proof of Theorem 11. It can be verified that:

$$
(\{x, y\} \subseteq I) \wedge(x \neq y) \Rightarrow \text { childset }(x) \cap \operatorname{childset}(y)=\emptyset
$$

Also, let totalhops $(x)$ denote the total number of hops traveled by application messages that created non-quiescent intervals in childset $(x)$. From the definition of $I$,

$x \in I \Rightarrow$ totalhops $(x) \geqslant D$

We have,

$$
\begin{aligned}
& \{\text { using }(9)\} \\
& \sum_{x \in I} \operatorname{totalhops}(x) \leqslant M H \\
\Rightarrow \quad & \{\text { using }(10)\} \\
& D \times|I| \leqslant \sum_{x \in I} \text { totalhops }(x) \leqslant M H \\
\Rightarrow \quad & \{\text { algebra }\} \\
& |I| \leqslant M H / D
\end{aligned}
$$

This establishes the theorem.

Note that, to achieve optimality, our algorithm does not require the knowledge of $H$, the average number of hops traveled by application messages. Also, as opposed to our algorithm, Mahapatra and Dutt's algorithm [21], which is also latency-optimal, has worst-case message complexity of $O(M D+N)$ irrespective of the average number of hops traveled by application messages. Therefore Mahapatra and Dutt's algorithm [21] is message-optimal only for those computations for which $H$ is $\Theta(D)$. On the other hand, our algorithm is message-optimal for all computations.

\section{Conclusion and future work}

In this paper, we have presented three algorithms for termination detection when processes and channels are reliable, and all channels are bidirectional. All three of the algorithms have optimal message complexity under varying assumptions. Moreover, they have optimal detection latency when message processing time is ignored. Algorithms TDA-SI and TDA-SI-MH have to be initiated along with the computation. The former algorithm is optimal when application messages are only exchanged between neighboring processes, whereas the latter is optimal when application messages can be exchanged between arbitrary processes. Algorithm TDA-DI can be initiated at any time after the computation has started. However, all channels are required to be FIFO for the algorithm to work correctly, which is also necessary to solve the problem.

All of our algorithms have three limitations currently. First, all processes need to know the diameter of the communication topology within a constant factor. (It is not necessary to know the exact value of the diameter as long as the estimate is within a constant factor of the actual value.) Second, they are asymmetric in the sense that one of the processes acts as a coordinator and is responsible for maintaining the state of the system. Third, our algorithms have optimal detection latency only when message processing time is ignored. An interesting (but challenging) research problem is to design a message-optimal termination detection algorithm that has optimal detection latency even when message processing time is explicitly considered, is fully symmetric, and in which the amount of knowledge a process needs to have about the system is minimized [22].

Acknowledgements We would like to thank the anonymous referees for their numerous and valuable suggestions, which greatly helped us in presenting the paper in a clear manner.

\section{References}

1. Alagar, S., Venkatesan, S.: An optimal algorithm for recording snapshots using casual message delivery. Inform. Process. Lett. 50, 311-316 (1994)

2. Atreya, R., Mittal, N., Garg, V. K.: Detecting locally stable predicates without modifying application messages. In: Proceedings 
of the 7th International Conference on Principles of Distributed Systems (OPODIS), pp. 20-33, La Martinique, France (2003)

3. Attiya, H., Welch, J.: Distributed Computing: Fundamentals, Simulations and Advanced Topics. 2nd ed. Wiley, New York (2004)

4. Chandrasekaran, S., Venkatesan, S.: A message-optimal algorithm for distributed termination detection. J. Parallel Distrib. Comput. 8(3), 245-252 (1990)

5. Chandy, K.M., Lamport, L.: Distributed snapshots: determining global states of distributed systems. ACM Trans. Comput. Syst. 3(1), 63-75 (1985)

6. Chandy, K.M., Misra, J.: How processes learn. Distrib. Comput. 1(1), 40-52 (1986)

7. Dally, W.J.: Performance analysis of $k$-ary- $n$ cube interconnection networks. IEEE Trans. Comput. 39(6), 775-785 (1990)

8. Demirbas, M., Arora, A.: An Optimal Termination Detection Algorithm for Rings. Technical Report OSU-CISRC-2/00-TR05, The Ohio State University (2000)

9. Dijkstra, E.W.: Shmuel Safra's Version of Termination Detection. EWD Manuscript 998. Available at http://www.cs.utexas. edu/users/EWD/, (1987)

10. Dijkstra, E.W., Feijen, W.H.J., van Gasteren, A.J.M.: Derivation of a termination detection algorithm for distributed computations. Inform. Process. Lett. 16, 217-219 (1983)

11. Dijkstra, E.W., Scholten, C.S.: Termination detection for diffusing computations. Inform. Process. Lett. 11(1), 1-4 (1980)

12. Francez, N.: Distributed termination. ACM Trans. Program. Lang. Syst. 2(1), 42-55 (1980)

13. Hélary, J.-M., Jard, C., Plouzeau, N., Raynal, M.: Detection of stable properties in distributed applications. In: Proceedings of the ACM Symposium on Principles of Distributed Computing (PODC), pp. 125-136 (1987)

14. Hélary, J.-M., Raynal, M.: Towards the construction of distributed detection programs, with an application to distributed termination. Distrib. Comput. 7(3), 137-147 (1994)

15. Huang, S.-T.: Detecting termination of distributed computations by external agents. In: Proceedings of the IEEE International Conference on Distributed Computing Systems (ICDCS), pp. 79-84 (1989)

16. Huang, S.-T.: Termination detection by using distributed snapshots. Inform. Process. Lett. 32, 113-119 (1989)

17. Lai, T.-H., Tseng, Y.-C., Dong, X.: A more efficient messageoptimal algorithm for distributed termination detection. In: Proceedings of the 6th International Parallel and Processing Symposium (IPPS), pp. 646-649. IEEE Computer Society (1992)
18. Lai, T.-H., Yang, T.H.: On distributed snapshots. Inform. Process. Lett. 25(3), 153-158 (1987)

19. Lamport, L.: Time, clocks, and the ordering of events in a distributed system. Commun. ACM 21(7), 558-565 (1978)

20. Lynch, N.: Distributed Algorithms. Morgan Kaufmann, (1996)

21. Mahapatra, N.R., Dutt, S.: An efficient delay-optimal distributed termination detection algorithm. J. Parallel Distrib. Comput. (2004) (to appear)

22. Matocha, J., Camp, T.: A taxonomy of distributed termination detection algorithms. J. Syst. Softw. 43(3), 207-221 (1998)

23. Mattern, F.: Algorithms for distributed termination detection. Distrib. Comput. 2(3), 161-175 (1987)

24. Mattern, F.: Global quiescence detection based on credit distribution and recovery. Inform. Process. Lett. 30(4), 195-200 (1989)

25. Mattern, F., Mehl, H., Schoone, A., Tel, G.: Global virtual time approximation with distributed termination detection algorithms, Technical Report RUU-CS-91-32. University of Utrecht, The Netherlands (1991)

26. Misra, J.: Detecting termination of distributed computations using markers. In: Proceedings of the ACM Symposium on Principles of Distributed Computing (PODC), pp. 290-294 (1983)

27. Mittal, N., Venkatesan, S., Peri, S.: Message-optimal and latencyoptimal termination detection algorithms for arbitrary topologies. In: Proceedings of the 18th Symposium on Distributed Computing (DISC), pp. 290-304, Amsterdam, The Netherlands (2004)

28. Peri, S., Mittal, N.: On termination detection in an asynchronous distributed system. In: Proceedings of the ISCA International Conference on Parallel and Distributed Computing Systems (PDCS), pp. 209-215, San Francisco, CA, USA (2004)

29. Rana, S.P.: A distributed solution of the distributed termination problem. Inform. Process. Lett. 17(1), 43-46 (1983)

30. Shavit N., Francez N. (1986) A new approach to detection of locally indicative stability. In: Proceedings of the International Colloquium on Automata, Languages and Systems (ICALP), pp. 344358, Rennes, France

31. Tel, G.: Introduction to Distributed Algorithms. 2nd ed. Cambridge University Press (US Server), Cambridge (2000)

32. Tel, G., Mattern, F.: The derivation of distributed termination detection algorithms from garbage collection schemes. ACM Trans. Programm. Lang. Syst. 15(1), 1-35 (1993) 